\title{
GLOBAL EXISTENCE RESULTS FOR OLDROYD-B FLUIDS IN EXTERIOR DOMAINS: THE CASE OF NON-SMALL COUPLING PARAMETERS
}

\author{
DAOYUANG FANG, MATTHIAS HIEBER AND RUIZHAO ZI
}

\begin{abstract}
Consider the set of equations describing Oldroyd-B fluids in an exterior domain. It is shown that this set of equations admits a unique, global solution in a certain function space provided the initial data, but not necessarily the coupling constant, is small enough.
\end{abstract}

\section{INTRODUCTION}

The theory of Oldroyd-B fluids recently gained quite some attention. This type of fluids is described by the following set of equations

$$
\left\{\begin{aligned}
\operatorname{Re}\left(\partial_{t} u+(u \cdot \nabla) u\right)-(1-\alpha) \Delta u+\nabla p & =\operatorname{div} \tau & & \text { in } \Omega \times(0, T), \\
\operatorname{div} u & =0 & & \text { in } \Omega \times(0, T), \\
W e\left(\partial_{t} \tau+(u \cdot \nabla) \tau+g_{a}(\tau, \nabla u)\right)+\tau & =2 \alpha D(u) & & \text { in } \Omega \times(0, T), \\
u & =0 & & \text { on } \partial \Omega \times(0, T), \\
u(0) & =u_{0} & & \text { in } \Omega, \\
\tau(0) & =\tau_{0} & & \text { in } \Omega,
\end{aligned}\right.
$$

where $\Omega \subset \mathbb{R}^{3}$ is a domain, $T \in(0, \infty]$ and $R e$ and $W e$ denote the Reynolds and Weissenberg number of the fluid, respectively. Moreover, the term $g_{a}$ is given by $g_{a}(\tau, \nabla u):=\tau W(u)-W(u) \tau-$ $a(D(u) \tau+\tau D(u))$ for some $a \in[-1,1]$, where $D(u)=1 / 2\left(\nabla u+(\nabla u)^{\top}\right)$ denotes the deformation tensor and $W(u)=1 / 2\left(\nabla u-(\nabla u)^{\top}\right)$ the vorticity tensor, respectively.

This set of equations originally was introduced by J.G. Oldroyd [17, whose intention it was to describe mathematically viscoelastic effects of certain types of fluids.

The study of the above set of equations started by a pioneering paper by Guillopé and Saut in 1990, see [5], who proved for the situation of bounded domains $\Omega \subset \mathbb{R}^{3}$, the existence of a local, strong solution to equation (1.1) in suitable Sobolev spaces $H^{s}(\Omega)$. Moreover, this solution exists on $[0, \infty)$ provided the data as well as the coupling constant $\alpha$ between the two equations are sufficiently small. For extensions to this results to the $L^{p}$-setting, see the work of Fernandéz-Cara, Guillén and Ortega [2].

The existence of global weak solutions in the case of $\Omega=\mathbb{R}^{n}$ was proved by Lions and Masmoudi in [15] for $a=0$. For extensions of this result to scaling invariant spaces of the form $L_{\mathrm{loc}}^{\infty}\left([0, T) ; H^{s}\left(\mathbb{R}^{3}\right)\right)$ for $s>3 / 2$, we refer to the work of Chemin and Masmoudi [1]. An improvement of the Chemin-Masmoudi blow-up criterion was presented recently by Lei, Masmoudi and Zhou in [11.

The situation of infinite Weissenberg numbers, mainly using the Lagrangian setting, was considered for $\Omega=\mathbb{R}^{3}$ or for bounded domains $\Omega \subset \mathbb{R}^{3}$ by Lin, Liu and Zhang in [13, Lei, Liu and Zhou in [10] and Lin and Zhang in 14. Further results, describing in particular the two dimensional situation, can be found in in [12, [8] and [9].

2000 Mathematics Subject Classification. 35Q35,76D03,76D05.

Key words and phrases. Oldroyd-B fluids, exterior domains, global solution. 
The situation of exterior domains was considered first in [6]. There, the existence of a unique, global solution defined in certain function spaces was proved provided the initial data as well as the coupling constant $\alpha$ are small enough.

In this paper we improve the main result given in [6] to the situation of non-small coupling coefficients $\alpha$. Indeed, given an exterior domain $\Omega \subset \mathbb{R}^{3}$ with smooth boundary, we prove the existence of a unique, global solution to equation (1.1) only under the assumption that the intial data $u_{0}$ and $\tau_{0}$ are sufficiently small in their natural norms. The idea of our approach is not completely new and goes back to the work of Molinet and Talhouk [16]. The main idea is to take the divergence in the third equation in (1.1) and to control $\mathbb{P} \operatorname{div} \tau$ in the $H^{1}$-norm by the corresponding term in $L^{2}$ and by $\operatorname{curl} \operatorname{div} \tau$, also measured in the $L^{2}$-norm. For doing this, we need in particular to extend the well known estimate for the $H^{1}$-norm of a function $u$ by the $L^{2}$-norms of $u, \operatorname{div} u, \operatorname{curl} u$ and by $u \cdot \nu$ in the $H^{1 / 2}$-norm for bounded domains to the situation of unbounded domains. Note that the main difficulty in the case of exterior domains is due to the failure of Poincaré's inequality in this situation. Hence, we must treat the lower order terms of $u$ and $\partial_{t} u$ in a new way and some new higher order energy estimates for $u$ and $\partial_{t} u$ need to be developed as well.

These higher order energy estimates then imply that the local solution is satisfying a certain differential inequality which in turn implies that the local solution of (1.1) can be extended for all positive times.

It is worthwhile to mention that also our approach to obtain local solutions to (1.1) seems to be new and quite different from the ones known in the literature. In fact, we base our local existence argument not on Schauder's fixed point theorem as it has been done in almost all of the existing works, but on a variant of Banach's contraction principle, avoiding hereby unnecessary uniqueness arguments.

Some words about the derivation of equation (1.1) are in order. In fact, incompressible fluids are subject to the following system of equations

$$
\left\{\begin{aligned}
\partial_{t} u+(u \cdot \nabla) u & =\operatorname{div} \sigma \\
\operatorname{div} u & =0,
\end{aligned}\right.
$$

where $u, \sigma$ are the velocity and stress tensor, respectively. Moreover, $\sigma$ can be decomposed into $-p I d+\tau$, where $p$ denotes the pressure of the fluid and $\tau$ the tangential part of the stress tensor, respectively. For the Oldroyd-B model, $\tau$ is given by the relation

$$
\tau+\lambda_{1} \frac{D_{a} \tau}{D t}=2 \eta\left(D(u)+\lambda_{2} \frac{D_{a} D(u)}{D t}\right)
$$

where $\frac{D_{a}}{D t}$ denotes the "objective derivative"

$$
\frac{D_{a} \tau}{D t}=\partial_{t} \tau+(u \cdot \nabla) \tau+g_{a}(\tau, \nabla u)
$$

and $g_{a}(\tau, \nabla u):=\tau W(u)-W(u) \tau-a(D(u) \tau+\tau D(u))$ for some $a \in[-1,1]$. Here $D(u)$ is the deformation tensor defined as above and $W(u)$ denotes the vorticity tensor. The parameters $\lambda_{1}>\lambda_{2}>0$ denote the relaxation and retardation time, respectively.

The tangential part of the stress tensor $\tau$ can be decomposed as $\tau=\tau_{N}+\tau_{e}$ where $\tau_{N}=2 \eta \frac{\lambda_{2}}{\lambda_{1}} D(u)$ corresponds to the Newtonian part and $\tau_{e}$ to the purely elastic part. Here $\eta$ denotes the fluid viscosity. Denoting $\tau_{e}=\tau$, the above equations can be rewritten as

$$
\left\{\begin{aligned}
\partial_{t} u+(u \cdot \nabla) u-\eta(1-\alpha) \Delta u+\nabla p & =\operatorname{div} \tau, \\
\operatorname{div} u & =0 \\
\tau+\lambda_{1} \frac{D_{a} \tau}{D t} & =2 \eta \alpha D(u),
\end{aligned}\right.
$$

with the retardation parameter $\alpha:=1-\frac{\lambda_{2}}{\lambda_{1}} \in(0,1)$.

Using dimensionless variables, Oldroyd-B fluids may be thus described by the equations (1.1). 
In order to formulate our main result, let $A$ be the Stokes operator in $L_{\sigma}^{2}(\Omega)$ defined by

$$
A u:=-\mathbb{P} \Delta u \text { for all } u \in D(A):=H^{2}(\Omega) \cap H_{0}^{1}(\Omega) \cap L_{\sigma}^{2}(\Omega),
$$

where the space $L_{\sigma}^{2}(\Omega)$ is defined precisely later in Section 2. Moreover, we set $V:=H_{0}^{1}(\Omega) \cap L_{\sigma}^{2}(\Omega)$.

Our main results then reads as follows.

Theorem 1.1. Let $\Omega \subset \mathbb{R}^{3}$ be an exterior domain with boundary $\partial \Omega$ of class $C^{3}$ and let $J=[0, \infty)$. Then there exists $\varepsilon_{0}>0$ such that if

$$
\left\|u_{0}\right\|_{D(A)}+\left\|\tau_{0}\right\|_{H^{2}(\Omega)} \leq \varepsilon_{0},
$$

then equation (1.1) admits a unique, global strong solution $(u, p, \tau)$ for all $t \in J$ satisfying

$$
\begin{aligned}
u & \in C_{b}(J ; D(A)) \text { with } \nabla u \in L^{2}\left(J ; H^{2}\right) \text { and } u^{\prime} \in L^{2}(J ; V) \cap C_{b}\left(J ; L_{\sigma}^{2}(\Omega)\right), \\
\nabla p & \in L^{2}\left(J ; H^{1}(\Omega)\right) \cap L^{\infty}\left(J ; H^{1}(\Omega)\right), \\
\tau & \in C_{b}\left(J ; H^{2}(\Omega)\right) \cap L^{2}\left(J ; H^{2}(\Omega)\right) \text { with } \tau^{\prime} \in C_{b}\left(J ; H^{1}(\Omega)\right) \cap L^{2}\left(J ; L^{2}(\Omega)\right) .
\end{aligned}
$$

\section{Preliminaries}

We start this section by recalling a higher order elliptic regularity estimate for the stationary Stokes equations. A proof can be found for example in [3], Theorem $\mathbf{V}$ 4.7. In the following, we denote by $\hat{H}^{k}(\Omega)$ the homogeneous Sobolev spaces of order $k$.

Lemma 2.1. Let $m \in\{0,1\}$ and $n \geq 3$. Assume that $\Omega \subset \mathbb{R}^{n}$ is an exterior domain with boundary of class $C^{m+2}$ and $g \in H^{m}(\Omega)$. Then the equation

$$
\left\{\begin{aligned}
-\Delta u+\nabla p=g & \text { in } \Omega, \\
\operatorname{div} u=0 & \text { in } \Omega, \\
u=0 & \text { on } \partial \Omega
\end{aligned}\right.
$$

admits a solution $(u, p) \in \hat{H}^{m+2}(\Omega) \times \hat{H}^{m+1}(\Omega)$ which is unique provided $\nabla u \in L^{2}(\Omega)$. In this case, there exists a constants $C>0$ such that

$$
\left\|\nabla^{2} u\right\|_{H^{m}}+\|\nabla p\|_{H^{m}} \leq C\left(\|g\|_{H^{m}}+\|\nabla u\|_{L^{2}}\right) .
$$

The following variant of Lemma 2.1 will be important in the sequel. We first introduce the spaces

$$
G_{2}(\Omega):=\left\{u \in L^{2}(\Omega): u=\nabla \pi \text { for some } \pi \in H_{l o c}^{1}(\Omega)\right\},
$$

and

$$
L_{\sigma}^{2}(\Omega):=\left\{u \in C_{c}^{\infty}(\Omega): \operatorname{div} u=0 \text { in } \Omega\right\}^{-\|\cdot\|_{L^{2}}}
$$

Then $L^{2}(\Omega)$ can be decomposed into

$$
L^{2}(\Omega)=L_{\sigma}^{2}(\Omega) \oplus G_{2}(\Omega),
$$

and there exists a unique projection $\mathbb{P}: L^{2}(\Omega) \rightarrow L_{\sigma}^{2}(\Omega)$ with $G_{2}(\Omega)$ as its null space. $\mathbb{P}$ is called the Helmholtz projection. It is well known that

$$
L_{\sigma}^{2}(\Omega)=\left\{u \in L^{2}(\Omega): \operatorname{div} u=0,\left.\nu \cdot u\right|_{\partial \Omega}=0\right\},
$$

where $\nu$ is the exterior normal to $\partial \Omega$.

Corollary 2.2. Assume that the assumptions of Lemma 2.1 hold. Then there exists a constant $C>0$ such that the solution $(u, p)$ of equation (2.1) satisfies

$$
\left\|\nabla^{2} u\right\|_{H^{m}} \leq C\left(\|\mathbb{P} g\|_{H^{m}}+\|\nabla u\|_{L^{2}}\right) .
$$


DAOYUANG FANG, MATTHIAS HIEBER AND RUIZHAO ZI

Proof. Applying the Helmholtz decomposition to $g$ and noting that $\mathbb{P}$ acts as a bounded operator on $H^{1}(\Omega)$ yields $g=\mathbb{P} g+\nabla \varphi$ for some $\nabla \varphi \in H^{m}(\Omega)$. Hence, the first line of the above Stokes equation may be rewritten as $-\Delta u+\nabla \tilde{p}=\mathbb{P} g$, where $\tilde{p}=p-\varphi$. Thus, estimate (2.2) follows immediately from eestimate (2.1).

Remark 2.3. Rewriting the above Stokes equation (2.1) as $A u=\mathbb{P} g$, we may replace the term $\mathbb{P} g$ in (2.2) by $A u$.

We next recall a well known estimates for the $H^{1}$-norm of a function defined on a bounded domain $\Omega \subset \mathbb{R}^{3}$.

Lemma 2.4 (4]). Let $\Omega \subset \mathbb{R}^{3}$ be a bounded domain with boundary $\partial \Omega$ of class $C^{2}$. Then there exists a constant $C>0$ such that

$$
\|u\|_{H^{m}(\Omega)} \leq C\left(\|u\|_{L^{2}(\Omega)}+\|\operatorname{div} u\|_{L^{2}(\Omega)}+\|\operatorname{curl} u\|_{L^{2}(\Omega)}+\|u \cdot \nu\|_{H^{\frac{1}{2}(\partial \Omega)}}\right), \quad u \in H^{1}(\Omega)^{3} .
$$

The following variant of Lemma 2.4 concerns exterior domains. More precisely, the following proposition holds true.

Proposition 2.5. Let $\Omega \subset \mathbb{R}^{3}$ be an exterior domain with boundary $\partial \Omega$ of class $C^{2}$. Then there exists a constant $C>0$ such that

$$
\|u\|_{H^{1}(\Omega)} \leq C\left(\|u\|_{L^{2}(\Omega)}+\|\operatorname{div} u\|_{L^{2}(\Omega)}+\|\operatorname{curl} u\|_{L^{2}(\Omega)}+\|u \cdot \nu\|_{H^{\frac{1}{2}(\partial \Omega)}}\right), \quad u \in H^{1}(\Omega)^{3} .
$$

Proof. We establish first a similar inequality for functions defined on $\mathbb{R}^{3}$. Assuming, for the time being, that $u$ is smooth, we obtain for the $j$-th component $u^{j}$ of $u$

$$
\Delta u^{j}=\sum_{1 \leq i \leq 3} \partial_{i}\left(\partial_{i} u^{j}-\partial_{j} u^{i}\right)+\partial_{j} \operatorname{div} u=\sum_{1 \leq i \leq 3} \partial_{i}(\operatorname{curl} u)^{i j}+\partial_{j} \operatorname{div} u .
$$

Hence,

and

$$
u^{j}=-\sum_{1 \leq i \leq 3} \partial_{i}(-\Delta)^{-1}(\operatorname{curl} u)^{i j}-\partial_{j}(-\Delta)^{-1} \operatorname{div} u,
$$

$$
\begin{aligned}
\partial_{k} u^{j} & =-\sum_{1 \leq i \leq 3} \partial_{k} \partial_{i}(-\Delta)^{-1}(\operatorname{curl} u)^{i j}-\partial_{j} \partial_{k}(-\Delta)^{-1} \operatorname{div} u \\
& =-\sum_{1 \leq i \leq 3} R_{i} R_{k}(\operatorname{curl} u)^{i j}-R_{j} R_{k} \operatorname{div} u
\end{aligned}
$$

where $R_{j}:=\partial_{j}(-\Delta)^{-\frac{1}{2}}$ denotes the $j$-th Riesz transforms. Classical results on the boundedness of the Riesz transforms imply

$$
\|\nabla u\|_{L^{2}} \leq C\left(\|\operatorname{div} u\|_{L^{2}}+\|\operatorname{curl} u\|_{L^{2}}\right)
$$

for some $C>0$. By density, this estimate transfers to functions $u$ belonging to $H^{1}\left(\mathbb{R}^{3}\right)$.

Next, our aim is to combine the above estimate with the corresponding one in bounded domains in order to obtain the assertion for exterior domains. To this end, let $R>0$ such that $\Omega^{c} \subset B_{R}(0):=\{x \in$ $\left.\mathbb{R}^{3}:|x|<R\right\}$ and set

$$
D:=\Omega \cap B_{R+3}(0), \quad \Gamma_{1}:=\partial \Omega \text { and } \Gamma_{2}:=\partial D \backslash \Gamma_{1} .
$$

Next, we choose a cut-off function $\phi \in C_{c}^{\infty}\left(B_{R+3}(0)\right)$ such that $0 \leq \phi \leq 1$ and

$$
\phi(x)= \begin{cases}1, & |x| \leq R+1 \\ 0, & |x| \geq R+2 .\end{cases}
$$

and decompose $u$ as

$$
u=\phi u+(1-\phi) u=: u_{1}+u_{2} .
$$


Clearly, $u_{1} \in H^{1}(D)$ and $u_{1} \equiv 0$ near $\Gamma_{2}$. Moreover, $u_{2} \in H_{0}^{1}(\Omega)$ and after zero extension (still denoted by $u_{2}$ ), we can regard $u_{2}$ as an element in $H^{1}\left(\mathbb{R}^{3}\right)$. Now it follows from (2.4) and (2.3) that

$$
\begin{aligned}
& \|\nabla u\|_{L^{2}(\Omega)} \leq\left\|\nabla u_{1}\right\|_{L^{2}(D)}+\left\|\nabla u_{2}\right\|_{L^{2}\left(\mathbb{R}^{3}\right)} \\
& \leq C\left[\|\phi u\|_{L^{2}(D)}+\|\operatorname{div}(\phi u)\|_{L^{2}(D)}+\|\operatorname{curl}(\phi u)\|_{L^{2}(D)}+\|(\phi u) \cdot \nu\|_{H^{\frac{1}{2}}\left(\Gamma_{1}\right)}\right. \\
& \left.+\|(\phi u) \cdot \nu\|_{H^{\frac{1}{2}\left(\Gamma_{2}\right)}}+\|\operatorname{div}((1-\phi) u)\|_{L^{2}\left(\mathbb{R}^{3}\right)}+\|\operatorname{curl}((1-\phi) u)\|_{L^{2}\left(\mathbb{R}^{3}\right)}\right] \\
& \leq C\left[\|u \cdot \nu\|_{H^{\frac{1}{2}\left(\Gamma_{1}\right)}}+\|\phi u\|_{L^{2}(D)}+\|\nabla \phi \cdot u\|_{L^{2}(D)}+\|\nabla \phi \otimes u-u \otimes \nabla \phi\|_{L^{2}(D)}\right. \\
& \left.+\|\phi \operatorname{div} u\|_{L^{2}(D)}+\|(1-\phi) \operatorname{div} u\|_{L^{2}\left(\mathbb{R}^{3}\right)}+\|\phi \operatorname{curl} u\|_{L^{2}(D)}+\|(1-\phi) \operatorname{curl} u\|_{L^{2}\left(\mathbb{R}^{3}\right)}\right] \\
& \leq C\left[\|u \cdot \nu\|_{H^{\frac{1}{2}\left(\Gamma_{1}\right)}}+\|u\|_{L^{2}(\Omega)}+\|\operatorname{div} u\|_{L^{2}(\Omega)}+\|\operatorname{curl} u\|_{L^{2}(\Omega)}\right],
\end{aligned}
$$

and the estimate (2.5) follows immediately.

In order to construct a local solution to (1.1), we study first two linearized equations; the first one for the velocity $u$ and the second one for the tangential part of the stress tensor $\tau$, respectively.

First, given $T>0$, we recall some results on the Stokes equation

$$
\left\{\begin{aligned}
\partial_{t} u-\Delta u+\nabla p & =f & & \text { in } \Omega \times(0, T), \\
\operatorname{div} u & =0 & & \text { in } \Omega \times(0, T), \\
u & =0 & & \text { on } \partial \Omega \times(0, T), \\
u(0) & =u_{0} & & \text { in } \Omega,
\end{aligned}\right.
$$

where $f$ is a given external force.

Proposition 2.6 ([18]). Let $\Omega \subset \mathbb{R}^{3}$ be an exterior domain with boundary $\partial \Omega$ of class $C^{3}$. Assume that $f \in L^{2}\left(0, T ; H^{1}(\Omega)\right), f^{\prime} \in L^{2}\left(0, T ; H^{-1}(\Omega)\right)$ and $u_{0} \in H^{2}(\Omega) \cap V(\Omega)$. Then there exists a unique solution $(u, p)$ of equation 2.4) satisfying

$$
\begin{aligned}
& u \in L^{2}\left(0, T ; H^{3}(\Omega)\right) \cap C\left([0, T] ; H^{2}(\Omega) \cap V(\Omega)\right), u^{\prime} \in L^{2}(0, T ; V(\Omega)) \cap C\left([0, T] ; L_{\sigma}^{2}(\Omega)\right), \\
& p \quad \in L^{2}\left(0, T ; H_{\text {loc }}^{2}(\Omega)\right) .
\end{aligned}
$$

Moreover, there exists a constant $C>0$ such that

$$
\begin{aligned}
\|u\|_{L^{2}\left(H^{3}\right) \cap L^{\infty}\left(H^{2} \cap V\right)}^{2}+\left\|u^{\prime}\right\|_{L^{2}(V) \cap L^{\infty}\left(L_{\sigma}^{2}\right)}^{2}+\|\nabla p\|_{L^{2}\left(H^{1}\right)}^{2} \leq & C\left[\left\|u_{0}\right\|_{H^{2}}^{2}+\|f(0)\|_{L^{2}}+\|f\|_{L^{2}\left(H^{1}\right)}\right. \\
& \left.+\left\|f^{\prime}\right\|_{L^{2}\left(H^{-1}\right)}\right] .
\end{aligned}
$$

Next, consider the transport equation

$$
\left\{\begin{aligned}
\text { We }\left(\partial_{t} \tau+(v \cdot \nabla) \tau\right)+\tau & =2 \alpha D(v)-\mathrm{We} g_{a}(\tau, \nabla v), & & \text { in } \Omega \times(0, T), \\
\tau(0) & =\tau_{0}, & & \text { in } \Omega,
\end{aligned}\right.
$$

where $v$ is a given velocity field.

Proposition 2.7 ([18]). Let $\Omega \subset \mathbb{R}^{3}$ be an exterior domain with boundary $\partial \Omega$ of class $C^{3}$. Assume that $v \in L^{1}\left(0, T ; H^{3}(\Omega) \cap V(\Omega)\right)$ and $\tau_{0} \in H^{2}(\Omega)$. Then there exists a unique solution of equation (2.5) and a constant $C>0$ such that

$$
\|\tau\|_{L^{\infty}\left(0, T ; H^{2}(\Omega)\right)} \leq\left(\left\|\tau_{0}\right\|_{H^{2}}+\frac{2 \alpha}{C W e}\right) \exp \left(C\|v\|_{L^{1}\left(H^{3}\right)}\right) .
$$

If, in addition, $v \in C\left([0, T] ; H^{2}(\Omega) \cap V(\Omega)\right)$, then $\tau^{\prime} \in C\left([0, T] ; H^{1}(\Omega)\right)$ and

$$
\left\|\tau^{\prime}\right\|_{L^{\infty}\left(0, T ; H^{1}\right)} \leq C\left(\|v\|_{L^{\infty}\left(H^{2}\right)}+\frac{1}{C W e}\right)\left(\left\|\tau_{0}\right\|_{H^{2}}+\frac{2 \alpha}{C W e}\right) \exp \left(C\|v\|_{L^{1}\left(H^{3}\right)}\right) .
$$

The assertions of Propositions 2.6 and 2.7 are stated e.g. in [18] even for a more general class of domains, however, without giving a proof. 


\section{Existence and Uniqueness of a Local Solution}

In this section we prove that the system (1.1) possesses a unique, local solution provided the intial data are smooth enough. More precisely, the following result holds true.

Proposition 3.1. Assume that $\Omega \subset \mathbb{R}^{3}$ is an exterior domain with boundary $\partial \Omega$ of class $C^{3}$. Let $u_{0} \in D(A)$ and $\tau_{0} \in H^{2}(\Omega)$. Then there exist $T_{\star}>0$ and functions

$$
\begin{aligned}
u & \in L^{2}\left(0, T_{\star} ; H^{3}(\Omega)\right) \cap C\left(\left[0, T_{\star}\right] ; D(A)\right) \text { with } u^{\prime} \in L^{2}\left(0, T_{\star}, V\right) \cap C\left(\left[0, T_{\star}\right] ; L_{\sigma}^{2}(\Omega)\right), \\
p & \in L^{2}\left(0, T ; H_{l o c}^{2}(\Omega)\right) \text { with } \nabla p \in L^{2}\left(0, T_{\star} ; H^{1}(\Omega)\right), \\
\tau & \in C\left(\left[0, T_{\star}\right] ; H^{2}(\Omega)\right) \text { with } \tau^{\prime} \in C\left(\left[0, T_{\star}\right], H^{1}(\Omega)\right)
\end{aligned}
$$

such that $(u, p, \tau)$ is the unique solution to equation (1.1) on $\left(0, T_{\star}\right)$.

Let us begin the proof of Proposition 3.1 with the following variant of Banach's fixed point theorem. For a proof, we refer e.g. to [7].

Lemma 3.2 ([7). Let $X$ be a reflexive Banach space or let $X$ have a separable pre-dual. Let $K$ be a convex, closed and bounded subset of $X$ and assume that $X$ is embedded into a Banach space $Y$. Let $\Phi: X \rightarrow X$ map $K$ into $K$ and assume there exists $q<1$ such that

$$
\|\Phi(x)-\Phi(x)\|_{Y} \leq q\|x-y\|_{Y}, \quad x, y \in K .
$$

Then there exists a unique fixed point of $\Phi$ in $K$.

Our proof of Proposition 3.1 relies on a combination of Propositions 2.6 and 2.7 with Lemma 3.2. To this end, consider for $T>0$ the following function spaces

$$
\begin{aligned}
E_{1} & :=L^{2}\left(0, T ; H^{3}(\Omega)\right) \cap L^{\infty}\left(0, T ; H^{2}(\Omega) \cap V\right), \\
E_{2} & :=L^{2}(0, T ; V(\Omega)) \cap L^{\infty}\left(0, T ; L_{\sigma}^{2}(\Omega)\right), \\
F_{1} & :=L^{\infty}\left(0, T ; H^{2}(\Omega)\right), \\
F_{2} & \left.:=L^{\infty}\left(0, T ; H^{1}(\Omega)\right)\right),
\end{aligned}
$$

and for $B_{1}, B_{2}>0$ define the set $K(T)$ by

$$
\begin{gathered}
K(T):=\left\{(v, \theta) \in E_{1} \times F_{1}, v^{\prime} \in E_{2}, \theta^{\prime} \in F_{2}, v(0)=u_{0}, \theta(0)=\tau_{0}\right. \text { and } \\
\left.\|v\|_{E_{1}}^{2}+\left\|v^{\prime}\right\|_{E_{2}}^{2} \leq B_{1},\|\theta\|_{F_{1}} \leq B_{1},\left\|\theta^{\prime}\right\|_{F_{2}} \leq B_{2}\right\} .
\end{gathered}
$$

Next, given $(v, \theta) \in K(T)$, we define the mapping

$$
\Phi(v, \theta):=(u, \tau)
$$

where $(u, \tau)$ is defined to be the unique solution of the corresponding linearized problem of (1.1)

$$
\left\{\begin{aligned}
\operatorname{Re} \partial_{t} u+(1-\alpha) A u & =-\mathbb{P} \operatorname{div}(v \otimes v)+\mathbb{P} \operatorname{div} \theta & & \text { in } \Omega \times(0, T), \\
\mathrm{We}\left(\partial_{t} \tau+(v \cdot \nabla) \tau\right)+\tau & =2 \alpha D(v)-\mathrm{We} g_{a}(\tau, \nabla v) & & \text { in } \Omega \times(0, T), \\
u & =0 & & \text { on } \partial \Omega \times(0, T), \\
u(0) & =u_{0} & & \text { in } \Omega, \\
\tau(0) & =\tau_{0} & & \text { in } \Omega,
\end{aligned}\right.
$$

where $A$ denotes the Stokes operator defined as in Section 1. It follows from Proposition 2.6 and 2.7 that for appropriate choices of $B_{1}$ and $B_{2}$, there exists $T_{1}>0$ such that $\Phi\left(K\left(T_{1}\right)\right) \subset K\left(T_{1}\right)$.

Next, we will prove that there exists $T_{\star} \in\left(0, T_{1}\right]$ such that $\Phi$ is contractive on $Y\left(T_{\star}\right)$, where $Y(T)$ is defined by

$$
Y(T):=\left\{(v, \theta) \in L^{\infty}\left(0, T ; L^{2}(\Omega)\right) \times L^{\infty}\left(0, T ; L^{2}(\Omega)\right), \nabla v \in L^{2}\left(0, T ; L^{2}(\Omega)\right)\right\} .
$$


Indeed, for $\left(v_{i}, \theta_{i}\right) \in K\left(T_{1}\right)$ let $\left(u_{i}, \tau_{i}\right)=\Phi\left(v_{i}, \theta_{i}\right)$ for $i=1,2$. Moreover, we set $\bar{u}=u_{1}-u_{2}$ and $\bar{\tau}=\tau_{1}-\tau_{2}$. Then $(\bar{u}, \bar{\tau})$ satisfies the equation

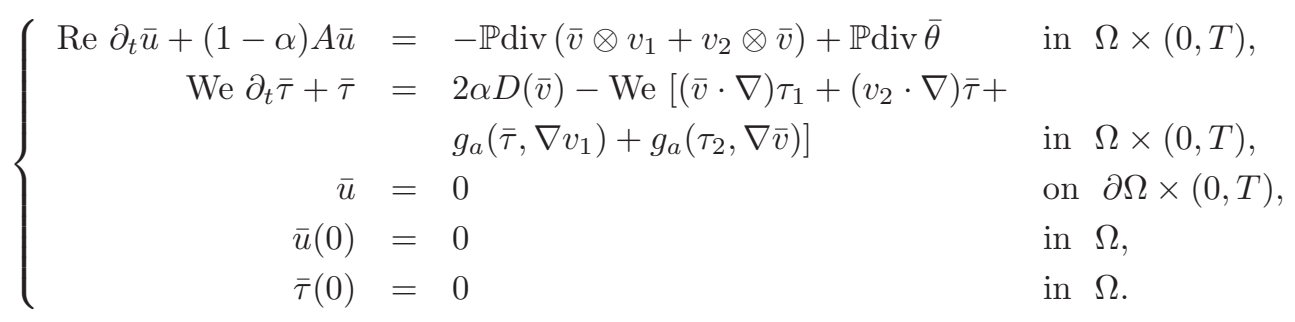

Taking the $L^{2}$ inner product of $(3.2)_{1}$ with $\bar{u}$, we obtain

$$
\begin{aligned}
\frac{1}{2} \frac{d}{d t}\left(\operatorname{Re}\|\bar{u}\|_{L^{2}}^{2}\right)+(1-\alpha)\|\nabla \bar{u}\|_{L^{2}}^{2} & =\operatorname{Re}\left(\bar{v} \otimes v_{1}+v_{2} \otimes \bar{v} \mid \nabla \bar{u}\right)-(\bar{\theta} \mid \nabla \bar{u}) \\
& \leq \frac{1-\alpha}{2}\|\nabla \bar{u}\|_{L^{2}}^{2}+\frac{2 \operatorname{Re}^{2}}{1-\alpha}\left(\left\|v_{1}\right\|_{L^{\infty}}^{2}+\left\|v_{2}\right\|_{L^{\infty}}^{2}\right)\|\bar{v}\|_{L^{2}}^{2}+\frac{1}{1-\alpha}\|\bar{\theta}\|_{L^{2}}^{2} \\
& \leq \frac{1-\alpha}{2}\|\nabla \bar{u}\|_{L^{2}}^{2}+\frac{C \operatorname{Re}^{2}}{1-\alpha}\left(\left\|v_{1}\right\|_{H^{2}}^{2}+\left\|v_{2}\right\|_{H^{2}}^{2}\right)\|\bar{v}\|_{L^{2}}^{2}+\frac{1}{1-\alpha}\|\bar{\theta}\|_{L^{2}}^{2} .
\end{aligned}
$$

Consequently,

$$
\frac{d}{d t}\left(\operatorname{Re}\|\bar{u}\|_{L^{2}}^{2}\right)+(1-\alpha)\|\nabla \bar{u}\|_{L^{2}}^{2} \leq \frac{C \operatorname{Re}^{2}}{1-\alpha}\left(\left\|v_{1}\right\|_{H^{2}}^{2}+\left\|v_{2}\right\|_{H^{2}}^{2}\right)\|\bar{v}\|_{L^{2}}^{2}+\frac{2}{1-\alpha}\|\bar{\theta}\|_{L^{2}}^{2} .
$$

Taking the $L^{2}$ inner product of $(\underline{3.2})_{2}$ with $\bar{\tau}$, we are led to

$$
\begin{aligned}
\frac{1}{2} \frac{d}{d t}\left(\mathrm{We}\|\bar{\tau}\|_{L^{2}}^{2}\right)+\|\bar{\tau}\|_{L^{2}}^{2}= & 2 \alpha(D(\bar{v}) \mid \bar{\tau})-\mathrm{We}\left((\bar{v} \cdot \nabla) \tau_{1}+g_{a}\left(\bar{\tau}, \nabla v_{1}\right)+g_{a}\left(\tau_{2}, \nabla \bar{v}\right) \mid \bar{\tau}\right) \\
\leq & \frac{\delta}{4}\|\nabla \bar{v}\|_{L^{2}}^{2}+\frac{4 \alpha^{2}}{\delta}\|\bar{\tau}\|_{L^{2}}^{2}+C \mathrm{We}\left(\|\bar{v}\|_{L^{6}}\left\|\nabla \tau_{1}\right\|_{L^{3}}+\|\nabla \bar{v}\|_{L^{2}}\left\|\tau_{2}\right\|_{L^{\infty}}\right)\|\bar{\tau}\|_{L^{2}} \\
& +C \mathrm{We}\left\|\nabla v_{1}\right\|_{L^{\infty}}\|\bar{\tau}\|_{L^{2}}^{2} \\
\leq & \frac{\delta}{4}\|\nabla \bar{v}\|_{L^{2}}^{2}+\frac{4 \alpha^{2}}{\delta}\|\bar{\tau}\|_{L^{2}}^{2}+C \mathrm{We}\|\nabla \bar{v}\|_{L^{2}}\left(\left\|\tau_{1}\right\|_{H^{2}}+\left\|\tau_{2}\right\|_{H^{2}}\right)\|\bar{\tau}\|_{L^{2}} \\
& +C \mathrm{We}\left\|\nabla v_{1}\right\|_{H^{2}}\|\bar{\tau}\|_{L^{2}}^{2} \\
\leq & \frac{\delta}{2}\|\nabla \bar{v}\|_{L^{2}}^{2}+\frac{C_{1}}{2}\left(\frac{1}{\delta}\left(1+\left\|\tau_{1}\right\|_{H^{2}}^{2}+\left\|\tau_{2}\right\|_{H^{2}}^{2}\right)+\left\|\nabla v_{1}\right\|_{H^{2}}\right) \mathrm{We}\|\bar{\tau}\|_{L^{2}}^{2},
\end{aligned}
$$

for some $C_{1}>0$ and all $\delta>0$. It follows from (3.3) and (3.4) that, for all $t \in[0, T]$,

$$
\begin{aligned}
\operatorname{Re}\|\bar{u}\|_{L^{2}}^{2}+ & \text { We }\|\bar{\tau}\|_{L^{2}}^{2}+\int_{0}^{t}\left((1-\alpha)\|\nabla \bar{u}\|_{L^{2}}^{2}+2\|\bar{\tau}\|_{L^{2}}^{2}\right) d s \\
\leq & T C_{2}\left(1+\left\|v_{1}\right\|_{L^{\infty}\left(H^{2}\right)}^{2}+\left\|v_{2}\right\|_{L^{\infty}\left(H^{2}\right)}^{2}\right)\left(\|\bar{v}\|_{L^{\infty}\left(L^{2}\right)}^{2}+\|\bar{\theta}\|_{L^{\infty}\left(L^{2}\right)}^{2}\right)+\delta \int_{0}^{t}\|\nabla \bar{v}\|_{L^{2}}^{2} d s \\
& +C_{1} \int_{0}^{t}\left(\frac{1}{\delta}\left(1+\left\|\tau_{1}\right\|_{H^{2}}^{2}+\left\|\tau_{2}\right\|_{H^{2}}^{2}\right)+\left\|\nabla v_{1}\right\|_{H^{2}}\right)\left(\mathrm{We}\|\bar{\tau}\|_{L^{2}}^{2}\right) d s \\
\leq & T C_{2}\left(1+2 B_{1}\right)\left(\|\bar{v}\|_{L^{\infty}\left(L^{2}\right)}^{2}+\|\bar{\theta}\|_{L^{\infty}\left(L^{2}\right)}^{2}\right)+\delta \int_{0}^{t}\|\nabla \bar{v}\|_{L^{2}}^{2} d s \\
& +C_{1} \int_{0}^{t}\left(\frac{1}{\delta}\left(1+\left\|\tau_{1}\right\|_{H^{2}}^{2}+\left\|\tau_{2}\right\|_{H^{2}}^{2}\right)+\left\|\nabla v_{1}\right\|_{H^{2}}\right)\left(\mathrm{We}\|\bar{\tau}\|_{L^{2}}^{2}\right) d s,
\end{aligned}
$$


for some $C_{2}>0$. Gronwall's inequality implies then

$$
\begin{aligned}
\operatorname{Re}\|\bar{u}\|_{L^{\infty}\left(L^{2}\right)}^{2}+ & \text { We }\|\bar{\tau}\|_{L^{\infty}\left(L^{2}\right)}^{2}+\int_{0}^{T}\left((1-\alpha)\|\nabla \bar{u}\|_{L^{2}}^{2}+2\|\bar{\tau}\|_{L^{2}}^{2}\right) d s \\
\leq & {\left[T C_{2}\left(1+2 B_{1}\right)\left(\|\bar{v}\|_{L^{\infty}\left(L^{2}\right)}^{2}+\|\bar{\theta}\|_{L^{\infty}\left(L^{2}\right)}^{2}\right)+\delta \int_{0}^{T}\|\nabla \bar{v}\|_{L^{2}}^{2} d s\right] } \\
& \times\left[1+C_{1}\left(\frac{T}{\delta}\left(1+\left\|\tau_{1}\right\|_{L^{\infty}\left(H^{2}\right)}^{2}+\left\|\tau_{2}\right\|_{\infty\left(H^{2}\right)}^{2}\right)+\sqrt{T}\left\|\nabla v_{1}\right\|_{L^{2}\left(H^{2}\right)}\right)\right. \\
& \left.\times \exp \left(C_{1}\left(\frac{T}{\delta}\left(1+\left\|\tau_{1}\right\|_{L^{\infty}\left(H^{2}\right)}^{2}+\left\|\tau_{2}\right\|_{\infty\left(H^{2}\right)}^{2}\right)+\sqrt{T}\left\|\nabla v_{1}\right\|_{L^{2}\left(H^{2}\right)}\right)\right)\right] \\
\leq & {\left[T C_{2}\left(1+2 B_{1}\right)\left(\|\bar{v}\|_{L^{\infty}\left(L^{2}\right)}^{2}+\|\bar{\theta}\|_{L^{\infty}\left(L^{2}\right)}^{2}\right)+\delta \int_{0}^{T}\|\nabla \bar{v}\|_{L^{2}}^{2} d s\right] } \\
& \times\left[1+C_{1}\left(\frac{T}{\delta}\left(1+2 B_{1}^{2}\right)+\sqrt{T B_{1}}\right) \exp \left(C_{1}\left(\frac{T}{\delta}\left(1+2 B_{1}^{2}\right)+\sqrt{\left.T B_{1}\right)}\right)\right]\right.
\end{aligned}
$$

Setting $\delta=\min \{\operatorname{Re}, \mathrm{We}, 1-\alpha\}\left(4+8 C_{1} \exp \left(2 C_{1}\right)\right)^{-1}$ and $T_{\star}=\min \left\{T_{1}, \frac{\delta}{1+2 B_{1}^{2}}, \frac{1}{B_{1}}, \frac{\min \{\operatorname{Re}, \mathrm{We}, 1-\alpha\}}{4 C_{2}\left(1+2 B_{1}\right)\left(1+2 C_{1} \exp \left(2 C_{1}\right)\right)}\right\}$, we see that for all $T \leq T_{\star}$,

$\|\bar{u}\|_{L^{\infty}\left(L^{2}\right)}^{2}+\|\bar{\tau}\|_{L^{\infty}\left(L^{2}\right)}^{2}+\int_{0}^{T}\left(\|\nabla \bar{u}\|_{L^{2}}^{2}+\|\bar{\tau}\|_{L^{2}}^{2}\right) d s \leq \frac{1}{4}\left(\|\bar{v}\|_{L^{\infty}\left(L^{2}\right)}^{2}+\|\bar{\theta}\|_{L^{\infty}\left(L^{2}\right)}^{2}+\int_{0}^{T}\|\nabla \bar{v}\|_{L^{2}}^{2} d s\right)$.

Hence, $\Phi$ is contractive as a mapping from $Y\left(T_{\star}\right)$ to $Y\left(T_{\star}\right)$. The assertion of Proposition 3.1 thus follows from Lemma 3.2

\section{Proof of the Main Theorem}

Let $(u, p, \tau)$ be the local solution to equation 1.1 constructed in Proposition 3.1 We recall from this proposition that

$$
\begin{aligned}
& u \in L^{2}\left(0, T_{\star} ; H^{3}\right) \cap C\left(\left[0, T_{\star}\right] ; D(A)\right) \text { with } u^{\prime} \in L^{2}\left(0, T_{\star} ; V\right) \cap C\left(\left[0, T_{\star}\right] ; L_{\sigma}^{2}\right) \text { and } \\
& \tau \in C\left(\left[0, T_{\star}\right] ; H^{2}\right) \text { with } \tau^{\prime} \in C\left(\left[0, T_{\star}\right] ; H^{1}\right) .
\end{aligned}
$$

Our proof for the existence of a unique, global solution to (1.1) is based on the following a priori estimates for $u, \tau, u^{\prime}$ and $\tau^{\prime}$.

Let us begin with an a priori estimate for $\tau$. To this end, we take the inner product of (1.1) 3 with $\tau$ and obtain

(4.1) $\frac{\mathrm{We}}{2} \frac{d}{d t}\|\tau\|_{L^{2}}^{2}+\|\tau\|_{L^{2}}^{2}=2 \alpha(D(u) \mid \tau)-\mathrm{We}\left(g_{a}(\tau, \nabla u) \mid \tau\right) \leq 2 \alpha\|\nabla u\|_{L^{2}}\|\tau\|_{L^{2}}+C \mathrm{We}\|\nabla u\|_{H^{2}}\|\tau\|_{L^{2}}^{2}$.

Similarly,

$$
\begin{aligned}
\frac{\mathrm{We}}{2} \frac{d}{d t}\|\nabla \tau\|_{L^{2}}^{2}+\|\nabla \tau\|_{L^{2}}^{2} & =2 \alpha(\nabla D(u) \mid \nabla \tau)-\mathrm{We}\left(\nabla g_{a}(\tau, \nabla u) \mid \nabla \tau\right)-\mathrm{We}\left(\partial_{l} u^{k} \partial_{k} \tau^{i j} \mid \partial_{l} \tau^{i j}\right) \\
& \leq 2 \alpha\left\|\nabla^{2} u\right\|_{L^{2}}\|\nabla \tau\|_{L^{2}}+C \text { We }\|\nabla u\|_{H^{2}}\|\nabla \tau\|_{L^{2}}^{2}+C \text { We }\left\|\nabla^{2} u\right\|_{L^{2}}\|\tau\|_{L^{\infty}}\|\nabla \tau\|_{L^{2}},
\end{aligned}
$$

and for $i, j, k, l, m \in\{1,2,3\}$

$$
\begin{aligned}
\frac{\mathrm{We}}{2} \frac{d}{d t}\left\|\nabla^{2} \tau\right\|_{L^{2}}^{2}+\left\|\nabla^{2} \tau\right\|_{L^{2}}^{2}= & 2 \alpha\left(\nabla^{2} D(u) \mid \nabla^{2} \tau\right)-\mathrm{We}\left(\partial_{l m} g_{a}(\tau, \nabla u)^{i j} \mid \partial_{l m} \tau^{i j}\right)-\mathrm{We}\left(\partial_{l m} u^{k} \partial_{k} \tau^{i j} \mid \partial_{l m} \tau^{i j}\right) \\
& -\mathrm{We}\left(\partial_{m} u^{k} \partial_{k l} \tau^{i j} \mid \partial_{l m} \tau^{i j}\right)-\mathrm{We}\left(\partial_{l} u^{k} \partial_{k m} \tau^{i j} \mid \partial_{l m} \tau^{i j}\right) \\
\leq & 2 \alpha\left\|\nabla^{3} u\right\|_{L^{2}}\left\|\nabla^{2} \tau\right\|_{L^{2}}+C \mathrm{We}\|\nabla u\|_{H^{2}}\left\|\nabla^{2} \tau\right\|_{L^{2}}^{2}+ \\
& C \mathrm{We}\left\|\nabla^{3} u\right\|_{L^{2}}\|\tau\|_{L^{\infty}}\left\|\nabla^{2} \tau\right\|_{L^{2}}+C \mathrm{We}\left\|\nabla^{2} u\right\|_{H^{1}}\|\nabla \tau\|_{H^{1}}\left\|\nabla^{2} \tau\right\|_{L^{2}} .
\end{aligned}
$$


Combing the above three inequalities, we obtain

$$
\begin{aligned}
\frac{\mathrm{We}}{2} \frac{d}{d t}\|\tau\|_{H^{2}}^{2}+\|\tau\|_{H^{2}}^{2} & \leq 2 \alpha\|\nabla u\|_{H^{2}}\|\tau\|_{H^{2}}+C \mathrm{We}\|\nabla u\|_{H^{2}}\|\tau\|_{H^{2}}^{2} \\
& \leq \frac{1}{2}\|\tau\|_{L^{2}}^{2}+C \alpha^{2}\|\nabla u\|_{H^{2}}^{2}+C \frac{\mathrm{We}^{2}}{\alpha^{2}}\|\tau\|_{H^{2}}^{4},
\end{aligned}
$$

and thus

$$
\text { We } \frac{d}{d t}\|\tau\|_{H^{2}}^{2}+\|\tau\|_{H^{2}}^{2} \leq C \alpha^{2}\|\nabla u\|_{H^{2}}^{2}+C \frac{\mathrm{We}^{2}}{\alpha^{2}}\|\tau\|_{H^{2}}^{4} .
$$

Before estimating $u$, let us first apply the Helmholz projection $\mathbb{P}$ to $(1.1)_{1}$. This yields

$$
\operatorname{Re}\left(\partial_{t} u+\mathbb{P}((u \cdot \nabla) u)\right)+(1-\alpha) A u=\mathbb{P} \operatorname{div} \tau .
$$

Next, we estimate the term $\|\nabla u\|_{H^{2}}$ appearing in the right hand side of (4.2). Corollary 2.2 and Remark 2.3 imply that

$$
\left\|\nabla^{2} u\right\|_{H^{1}} \leq C\left(\|A u\|_{H^{1}}+\|\nabla u\|_{L^{2}}\right) .
$$

We deduce from equation (4.3) that

$$
\begin{aligned}
\|\nabla A u\|_{L^{2}} & \leq \frac{\operatorname{Re}}{1-\alpha}\left\|\nabla u_{t}\right\|_{L^{2}}+\frac{\operatorname{Re}}{1-\alpha}\|\nabla \mathbb{P}((u \cdot \nabla) u)\|_{L^{2}}+\frac{1}{1-\alpha}\|\nabla \mathbb{P} \operatorname{div} \tau\|_{L^{2}} \\
& \leq \frac{\operatorname{Re}}{1-\alpha}\left\|\nabla u_{t}\right\|_{L^{2}}+\frac{\operatorname{Re}}{1-\alpha}\|(u \cdot \nabla) u\|_{H^{1}}+\frac{1}{1-\alpha}\|\nabla \mathbb{P} \operatorname{div} \tau\|_{L^{2}}
\end{aligned}
$$

By the Gagliardo-Nirenberg as well as by Sobolev's inequality, we have

$$
\|u\|_{L^{\infty}} \leq C\|u\|_{L^{6}}^{\frac{1}{2}}\|\nabla u\|_{L^{6}}^{\frac{1}{2}} \leq C\|\nabla u\|_{L^{2}}^{\frac{1}{2}}\|\nabla u\|_{H^{1}}^{\frac{1}{2}},
$$

which allows us to bound the term $\|(u \cdot \nabla) u\|_{H^{1}}$ as

$$
\begin{aligned}
\|(u \cdot \nabla) u\|_{H^{1}} & =\|\nabla((u \cdot \nabla) u)\|_{L^{2}}+\|(u \cdot \nabla) u\|_{L^{2}} \\
& \leq\|\nabla u\|_{L^{4}}^{2}+\|u\|_{L^{\infty}}\left\|\nabla^{2} u\right\|_{L^{2}}+\|u\|_{L^{6}}\|\nabla u\|_{L^{3}} \\
& \leq C\left(\|\nabla u\|_{H^{1}}^{2}+\|u\|_{L^{6}}^{\frac{1}{2}}\|\nabla u\|_{L^{6}}^{\frac{1}{2}}\left\|\nabla^{2} u\right\|_{L^{2}}+\|\nabla u\|_{L^{2}}^{\frac{3}{2}}\|\nabla u\|_{H^{1}}^{\frac{1}{2}}\right) \\
& \leq C\left(\|\nabla u\|_{H^{1}}^{2}+\|\nabla u\|_{L^{2}}^{\frac{1}{2}}\|\nabla u\|_{H^{1}}^{\frac{3}{2}}+\|\nabla u\|_{L^{2}}^{\frac{3}{2}}\|\nabla u\|_{H^{1}}^{\frac{1}{2}}\right) \\
& \leq C\|\nabla u\|_{H^{1}}^{2} .
\end{aligned}
$$

Recalling Corollary 2.2 and Remark 2.3. we infer that

$$
\|\nabla u\|_{H^{1}} \leq C\left(\|A u\|_{L^{2}}+\|\nabla u\|_{L^{2}}\right) .
$$

Combing now the estimates (4.4)-(4.7) with (4.8) yields

$$
\begin{aligned}
\|\nabla u\|_{H^{2}}^{2} \leq & C\left(\|A u\|_{L^{2}}^{2}+\|\nabla u\|_{L^{2}}^{2}+\frac{\operatorname{Re}^{2}}{(1-\alpha)^{2}}\left\|\nabla u_{t}\right\|_{L^{2}}^{2}+\frac{1}{(1-\alpha)^{2}}\|\nabla \mathbb{P} \operatorname{div} \tau\|_{L^{2}}^{2}\right. \\
& \left.+\frac{\operatorname{Re}^{2}}{(1-\alpha)^{2}}\|A u\|_{L^{2}}^{4}+\frac{\operatorname{Re}^{2}}{(1-\alpha)^{2}}\|\nabla u\|_{L^{2}}^{4}\right) .
\end{aligned}
$$

Finally, estimate (4.2) combined with estimate (4.9) implies that

$$
\begin{aligned}
\text { We } \frac{d}{d t}\|\tau\|_{H^{2}}^{2}+\|\tau\|_{H^{2}}^{2}+2\|\nabla u\|_{H^{2}}^{2} \leq & \kappa_{1}\left(\|A u\|_{L^{2}}^{2}+\|\nabla u\|_{L^{2}}^{2}+\left\|\nabla u_{t}\right\|_{L^{2}}^{2}+\|\nabla \mathbb{P} \operatorname{div} \tau\|_{L^{2}}^{2}\right) \\
& +C\left(\|A u\|_{L^{2}}^{4}+\|\tau\|_{H^{2}}^{4}+\|\nabla u\|_{L^{2}}^{4}\right),
\end{aligned}
$$

for some $\kappa_{1}>0$. 
Next, taking the inner product of (4.3) with $u$, we obtain

$$
\frac{\operatorname{Re}}{2} \frac{d}{d t}\|u\|_{L^{2}}^{2}+(1-\alpha)\|\nabla u\|_{L^{2}}^{2}=(\operatorname{div} \tau \mid u) .
$$

Adding this equation to equation (4.1), integrating by parts and using the fact that $\tau$ is symmetric, yields

$$
\begin{aligned}
\frac{1}{2} \frac{d}{d t}\left(\operatorname{Re}\|u\|_{L^{2}}^{2}+\frac{\mathrm{We}}{2 \alpha}\|\tau\|_{L^{2}}^{2}\right)+(1-\alpha)\|\nabla u\|_{L^{2}}^{2}+\frac{1}{2 \alpha}\|\tau\|_{L^{2}}^{2} & =-\frac{\mathrm{We}}{2 \alpha}\left(g_{a}(\tau, \nabla u) \mid \tau\right) \\
& \leq \frac{C \mathrm{We}}{2 \alpha}\|\tau\|_{L^{\infty}}\|\nabla u\|_{L^{2}}\|\tau\|_{L^{2}} \\
& \leq \frac{1-\alpha}{2}\|\nabla u\|_{L^{2}}^{2}+\frac{C \mathrm{We}^{2}}{(1-\alpha) \alpha^{2}}\|\tau\|_{H^{2}}^{4},
\end{aligned}
$$

which means that

$$
\frac{d}{d t}\left(\operatorname{Re}\|u\|_{L^{2}}^{2}+\frac{\mathrm{We}}{2 \alpha}\|\tau\|_{L^{2}}^{2}\right)+(1-\alpha)\|\nabla u\|_{L^{2}}^{2}+\frac{1}{\alpha}\|\tau\|_{L^{2}}^{2} \leq \frac{C \mathrm{We}^{2}}{(1-\alpha) \alpha^{2}}\|\tau\|_{H^{2}}^{4} .
$$

Taking the inner product of (4.3) with $A u$ yields

$$
\frac{\operatorname{Re}}{2} \frac{d}{d t}\|\nabla u\|_{L^{2}}^{2}+(1-\alpha)\|A u\|_{L^{2}}^{2} \leq \frac{1-\alpha}{2}\|A u\|_{L^{2}}^{2}+\frac{1}{1-\alpha}\|\mathbb{P} \operatorname{div} \tau\|_{L^{2}}^{2}+\frac{\operatorname{Re}^{2}}{1-\alpha}\|\mathbb{P}(u \cdot \nabla) u\|_{L^{2}}^{2} .
$$

Further, since

$$
\|\mathbb{P}(u \cdot \nabla) u\|_{L^{2}}^{2} \leq C\|(u \cdot \nabla) u\|_{L^{2}}^{2} \leq C\|u\|_{L^{6}}^{2}\|\nabla u\|_{L^{3}}^{2} \leq C\|\nabla u\|_{L^{2}}^{3}\|\nabla u\|_{H^{1}},
$$

it follows that

$$
\frac{d}{d t}\left(\operatorname{Re}\|\nabla u\|_{L^{2}}^{2}\right)+(1-\alpha)\|A u\|_{L^{2}}^{2} \leq \frac{2}{1-\alpha}\|\mathbb{P} \operatorname{div} \tau\|_{L^{2}}^{2}+\varepsilon\|\nabla u\|_{H^{1}}^{2}+\frac{C \operatorname{Re}^{4}}{\varepsilon(1-\alpha)^{2}}\|\nabla u\|_{L^{2}}^{6} .
$$

Similarly, taking the inner product of (4.3) with $u^{\prime}$, and using (4.12) once more, we see that

$$
\frac{d}{d t}\left((1-\alpha)\|\nabla u\|_{L^{2}}^{2}\right)+\operatorname{Re}\left\|u^{\prime}\right\|_{L^{2}}^{2} \leq \frac{2}{\operatorname{Re}}\|\operatorname{Pdiv} \tau\|_{L^{2}}^{2}+\varepsilon\|\nabla u\|_{H^{1}}^{2}+\frac{C \operatorname{Re}^{2}}{\varepsilon}\|\nabla u\|_{L^{2}}^{6}
$$

for $\varepsilon>0$. In view of (4.8), (4.13) and (4.14) and by choosing $\varepsilon$ small enough, we are led to

$$
\begin{aligned}
& \frac{d}{d t}\left((2 \operatorname{Re}+1-\alpha)\|\nabla u\|_{L^{2}}^{2}\right)+\operatorname{Re}\left\|\partial_{t} u\right\|_{L^{2}}^{2}+(1-\alpha)\|A u\|_{L^{2}}^{2} \\
\leq \quad & \kappa_{2}\left(\|\nabla u\|_{L^{2}}^{2}+\|\operatorname{Pdiv} \tau\|_{L^{2}}^{2}\right)+C\|\nabla u\|_{L^{2}}^{6},
\end{aligned}
$$

for some $\kappa_{2}>0$. Next, differentiating equations $(1.1)_{1}$ and $(1.1)_{3}$ with respect to $t$, and taking the inner product of the resulting equations with $\partial_{t} u$ and $\partial_{t} \tau$, respectively, we obtain

$$
\frac{\operatorname{Re}}{2} \frac{d}{d t}\left\|\partial_{t} u\right\|_{L^{2}}^{2}+(1-\alpha)\left\|\nabla u_{t}\right\|_{L^{2}}^{2}=\left(\operatorname{div} \tau_{t} \mid \partial_{t} u\right)-\operatorname{Re}\left(\left(u_{t} \cdot \nabla\right) u \mid \partial_{t} u\right),
$$

as well as

$$
\begin{aligned}
\frac{\mathrm{We}}{2} \frac{d}{d t}\left\|\partial_{t} \tau\right\|_{L^{2}}^{2}+\left\|\partial_{t} \tau\right\|_{L^{2}}^{2}= & 2 \alpha\left(D\left(u_{t}\right) \mid \partial_{t} \tau\right)-\mathrm{We}\left(\left(u_{t} \cdot \nabla\right) \tau \mid \partial_{t} \tau\right) \\
& -\mathrm{We}\left(g_{a}\left(\tau_{t}, \nabla u\right) \mid \tau_{t}\right)-\mathrm{We}\left(g_{a}\left(\tau, \nabla u_{t}\right) \mid \partial_{t} \tau\right) .
\end{aligned}
$$


It follows that

$$
\begin{aligned}
& \frac{1}{2} \frac{d}{d t}\left(\operatorname{Re}\left\|\partial_{t} u\right\|_{L^{2}}^{2}+\frac{\mathrm{We}}{2 \alpha}\left\|\partial_{t} \tau\right\|_{L^{2}}^{2}\right)+(1-\alpha)\left\|\nabla u_{t}\right\|_{L^{2}}^{2}+\frac{1}{2 \alpha}\left\|\partial_{t} \tau\right\|_{L^{2}}^{2} \\
\leq & C\|\nabla u\|_{H^{2}}\left(\operatorname{Re}\left\|\partial_{t} u\right\|_{L^{2}}^{2}+\frac{\mathrm{We}}{2 \alpha}\left\|\partial_{t} \tau\right\|_{L^{2}}^{2}\right)+\frac{\mathrm{We}}{2 \alpha}\left\|\partial_{t} u\right\|_{L^{6}}\|\nabla \tau\|_{L^{3}}\left\|\partial_{t} \tau\right\|_{L^{2}} \\
& +C \frac{\mathrm{We}}{2 \alpha}\left\|\nabla u_{t}\right\|_{L^{2}}\|\tau\|_{L^{\infty}}\left\|\partial_{t} \tau\right\|_{L^{2}} \\
\leq & C\|\nabla u\|_{H^{2}}\left(\operatorname{Re}\left\|\partial_{t} u\right\|_{L^{2}}^{2}+\frac{\mathrm{We}}{2 \alpha}\left\|\partial_{t} \tau\right\|_{L^{2}}^{2}\right)+C \frac{\mathrm{We}}{2 \alpha}\left\|\nabla u_{t}\right\|_{L^{2}}\|\tau\|_{H^{2}}\left\|\partial_{t} \tau\right\|_{L^{2}},
\end{aligned}
$$

and by Young's inequality that

$$
\begin{aligned}
& \frac{d}{d t}\left(\operatorname{Re}\left\|\partial_{t} u\right\|_{L^{2}}^{2}+\frac{\mathrm{We}}{2 \alpha}\left\|\partial_{t} \tau\right\|_{L^{2}}^{2}\right)+(1-\alpha)\left\|\nabla u_{t}\right\|_{L^{2}}^{2}+\frac{1}{\alpha}\left\|\partial_{t} \tau\right\|_{L^{2}}^{2} \\
\leq & \epsilon\|\nabla u\|_{H^{2}}^{2}+\frac{C}{\epsilon}\left(\operatorname{Re}^{2}\left\|\partial_{t} u\right\|_{L^{2}}^{4}+\frac{\mathrm{We}^{2}}{4 \alpha^{2}}\left\|\partial_{t} \tau\right\|_{L^{2}}^{4}\right)+\frac{C \mathrm{We}^{2}}{\alpha^{2}(1-\alpha)}\|\tau\|_{H^{2}}^{2}\left\|\partial_{t} \tau\right\|_{L^{2}}^{2} .
\end{aligned}
$$

Next, following an idea of Molinet and Talhouk [16], we estimate $\mathbb{P} \operatorname{div} \tau$ and $\operatorname{curl} \operatorname{div} \tau$, which will be then used in order to control $\|\mathbb{P} \operatorname{div} \tau\|_{H^{1}}$. In order to do so, we take the divergence of (1.1) 3 and, using the incompressible condition, we obtain

$$
\text { We }\left(\operatorname{div} \tau_{t}+\operatorname{div}((u \cdot \nabla) \tau)\right)+\operatorname{div} \tau+\text { We } \operatorname{div} g_{a}(\tau, \nabla u)=\alpha \Delta u
$$

which, together with the equation of $u$, implies that

$$
\frac{1-\alpha}{\alpha} \mathrm{We} \operatorname{div} \tau_{t}+\frac{1}{\alpha} \operatorname{div} \tau=-\frac{1-\alpha}{\alpha} \mathrm{We}\left[\operatorname{div}((u \cdot \nabla) \tau)+\operatorname{div} g_{a}(\tau, \nabla u)\right]+\operatorname{Re}\left[\partial_{t} u+(u \cdot \nabla) u\right]+\nabla p .
$$

Applying the Helmholtz projection $\mathbb{P}$ to this equation yields

$$
\frac{1-\alpha}{\alpha} \mathrm{We} \mathbb{P} \operatorname{div} \tau_{t}+\frac{1}{\alpha} \mathbb{P} \operatorname{div} \tau=\operatorname{Re} \partial_{t} u+\mathbb{P}\left[\operatorname{Re}(u \cdot \nabla) u-\frac{1-\alpha}{\alpha} \mathrm{We}\left(\operatorname{div}((u \cdot \nabla) \tau)+\operatorname{div} g_{a}(\tau, \nabla u)\right)\right] \text {. }
$$

Taking the inner product of the above equation with $\mathbb{P} \operatorname{div} \tau$ and integrating by parts, we deduce that

$$
\begin{gathered}
\frac{1-\alpha}{\alpha} \frac{\mathrm{We}}{2} \frac{d}{d t}\|\mathbb{P} \operatorname{div} \tau\|_{L^{2}}^{2}+\frac{1}{\alpha}\|\mathbb{P} \operatorname{div} \tau\|_{L^{2}}^{2}=-\operatorname{Re}\left(\nabla u_{t} \mid \tau\right)+\operatorname{Re}(\mathbb{P}(u \cdot \nabla) u \mid \mathbb{P} \operatorname{div} \tau) \\
-\frac{1-\alpha}{\alpha} \operatorname{We}(\mathbb{P} \operatorname{div}((u \cdot \nabla) \tau) \mid \mathbb{P} \operatorname{div} \tau)-\frac{1-\alpha}{\alpha} \operatorname{We}\left(\mathbb{P} \operatorname{div} g_{a}(\tau, \nabla u) \mid \mathbb{P} \operatorname{div} \tau\right) .
\end{gathered}
$$

Note that

$$
\begin{aligned}
\operatorname{Re}|(\mathbb{P}((u \cdot \nabla) u) \mid \mathbb{P} \operatorname{div} \tau)| & \leq \operatorname{Re}\|u\|_{L^{6}}\|\nabla u\|_{L^{3}}\|\tau\|_{H^{1}} \leq C \operatorname{Re}\|\nabla u\|_{L^{2}}\|\nabla u\|_{H^{1}}\|\tau\|_{H^{1}} \\
& \leq \frac{\varepsilon}{6}\|\nabla u\|_{H^{1}}^{2}+\frac{C \operatorname{Re}^{2}}{\varepsilon}\|\nabla u\|_{L^{2}}^{2}\|\tau\|_{H^{1}}^{2},
\end{aligned}
$$

and

$$
\begin{aligned}
\frac{1-\alpha}{\alpha} \mathrm{We}|(\mathbb{P} \operatorname{div}((u \cdot \nabla) \tau) \mid \mathbb{P} \operatorname{div} \tau)| & \leq C \frac{1-\alpha}{\alpha} \mathrm{We}\left(\left\|\left|\nabla u\left\|\nabla \tau\left|\left\|_{L^{2}}+\right\| u\left\|\nabla^{2} \tau \mid\right\|_{L^{2}}\right)\right\| \tau \|_{H^{1}}\right.\right.\right. \\
& \leq C \frac{1-\alpha}{\alpha} \mathrm{We}\left(\|\nabla u\|_{L^{6}}\|\nabla \tau\|_{L^{3}}+\|u\|_{L^{\infty}}\left\|\nabla^{2} \tau\right\|_{L^{2}}\right)\|\tau\|_{H^{1}} \\
& \leq C \frac{1-\alpha}{\alpha} \mathrm{We}\|\nabla u\|_{H^{1}}\|\tau\|_{H^{2}}^{2} \leq \frac{\varepsilon}{6}\|\nabla u\|_{H^{1}}^{2}+\frac{C \mathrm{We}^{2}(1-\alpha)^{2}}{\varepsilon \alpha^{2}}\|\tau\|_{H^{2}}^{4}
\end{aligned}
$$

where we have used the Gagliardo-Nirenberg inequality (4.6) again.

Moreover, a direct calculation yields

$$
\frac{1-\alpha}{\alpha} \mathrm{We}\left|\left(\mathbb{P} \operatorname{div} g_{a}(\tau, \nabla u) \mid \mathbb{P} \operatorname{div} \tau\right)\right| \leq \frac{\varepsilon}{6}\|\nabla u\|_{H^{1}}^{2}+\frac{C \mathrm{We}^{2}(1-\alpha)^{2}}{\varepsilon \alpha^{2}}\|\tau\|_{H^{2}}^{4} .
$$


which implies

(4.19)

$\frac{d}{d t}\left(\frac{1-\alpha}{\alpha} \mathrm{We}\|\mathbb{P} \operatorname{div} \tau\|_{L^{2}}^{2}\right)+\frac{2}{\alpha}\|\mathbb{P} \operatorname{div} \tau\|_{L^{2}}^{2} \leq \operatorname{Re}^{2}\left\|\nabla u_{t}\right\|_{L^{2}}^{2}+\|\tau\|_{L^{2}}^{2}+\varepsilon\|\nabla u\|_{H^{1}}^{2}+C_{\varepsilon}\left(\|\nabla u\|_{L^{2}}^{4}+\|\tau\|_{H^{2}}^{4}\right)$.

Applying the curl operator to the equation (4.17) we obtain

$$
\begin{aligned}
& \frac{1-\alpha}{\alpha} \text { We curl div } \tau_{t}+\frac{1}{\alpha} \operatorname{curl} \operatorname{div} \tau= \\
& \quad \operatorname{Re}\left[\operatorname{curl} u_{t}+\operatorname{curl}((u \cdot \nabla) u)\right]-\frac{1-\alpha}{\alpha} \operatorname{We}\left[\operatorname{curl} \operatorname{div}((u \cdot \nabla) \tau)+\operatorname{curl} \operatorname{div} g_{a}(\tau, \nabla u)\right] .
\end{aligned}
$$

Taking the inner product of this equation with $\operatorname{curl} \operatorname{div} \tau$ yields

$$
\begin{aligned}
& \frac{1-\alpha}{\alpha} \frac{\mathrm{We}}{2} \frac{d}{d t}\|\operatorname{curl} \operatorname{div} \tau\|_{L^{2}}^{2}+\frac{1}{\alpha}\|\operatorname{curl} \operatorname{div} \tau\|_{L^{2}}^{2} \leq \\
& \frac{1}{2 \alpha}\|\operatorname{curl} \operatorname{div} \tau\|_{L^{2}}^{2}+\frac{C \alpha \operatorname{Re}^{2}}{2}\left\|\nabla u_{t}\right\|_{L^{2}}^{2}+\operatorname{Re}(\operatorname{curl}((u \cdot \nabla) u) \mid \operatorname{curl} \operatorname{div} \tau)- \\
& \frac{1-\alpha}{\alpha} \mathrm{We}(\operatorname{curl} \operatorname{div}((u \cdot \nabla) \tau) \mid \operatorname{curl} \operatorname{div} \tau)-\frac{1-\alpha}{\alpha} \mathrm{We}\left(\operatorname{curl} \operatorname{div} g_{a}(\tau, \nabla u) \mid \operatorname{curl} \operatorname{div} \tau\right) .
\end{aligned}
$$

Noting that

$$
\begin{aligned}
{[\operatorname{curl} \operatorname{div}((u \cdot \nabla) \tau)]_{l j}=} & (u \cdot \nabla)(\operatorname{curl} \operatorname{div} \tau)_{l j}+\left(\partial_{l i} u^{k} \partial_{k} \tau^{i j}-\partial_{j i} u^{k} \partial_{k} \tau^{i l}\right) \\
& +\left(\partial_{i} u^{k} \partial_{k l} \tau^{i j}-\partial_{i} u^{k} \partial_{k j} \tau^{i l}\right)+\left(\partial_{l} u^{k} \partial_{k i} \tau^{i j}-\partial_{j} u^{k} \partial_{k i} \tau^{i l}\right),
\end{aligned}
$$

we obtain

$\frac{1-\alpha}{\alpha} \operatorname{We}|(\operatorname{curl} \operatorname{div}((u \cdot \nabla) \tau) \mid \operatorname{curl} \operatorname{div} \tau)| \leq C \frac{1-\alpha}{\alpha} \mathrm{We}\left(\left\|\nabla^{2} u\right\|_{L^{6}}\|\nabla \tau\|_{L^{3}}+\|\nabla u\|_{L^{\infty}}\left\|\nabla^{2} \tau\right\|_{L^{2}}\right)\left\|\nabla^{2} \tau\right\|_{L^{2}}$

Moreover,

$$
\leq \frac{\varepsilon}{6}\|\nabla u\|_{H^{2}}^{2}+\frac{C(1-\alpha)^{2} \mathrm{We}^{2}}{\varepsilon \alpha^{2}}\|\tau\|_{H^{2}}^{4} .
$$

$$
\begin{aligned}
\operatorname{Re}|(\operatorname{curl}((u \cdot \nabla) u) \mid \operatorname{curl} \operatorname{div} \tau)| & \leq C \operatorname{Re}\left(\left\||\nabla u|^{2}\right\|_{L^{2}}+\left\|\left|u\left\|\nabla^{2} u \mid\right\|_{L^{2}}\right)\right\| \nabla^{2} \tau \|_{L^{2}}\right. \\
& \leq C \operatorname{Re}\left(\|\nabla u\|_{L^{\infty}}\|\nabla u\|_{L^{2}}+\|u\|_{L^{6}}\left\|\nabla^{2} u\right\|_{L^{3}}\right)\left\|\nabla^{2} \tau\right\|_{L^{2}} \\
& \leq C \operatorname{Re}\|\nabla u\|_{H^{2}}\|\nabla u\|_{L^{2}}\|\tau\|_{H^{2}} \leq \frac{\varepsilon}{6}\|\nabla u\|_{H^{2}}^{2}+\frac{C \operatorname{Re}^{2}}{\varepsilon}\|\nabla u\|_{L^{2}}^{2}\|\tau\|_{H^{2}}^{2},
\end{aligned}
$$

and a direct computation yields

$$
\frac{1-\alpha}{\alpha} \operatorname{We}\left|\left(\operatorname{curl} \operatorname{div} g_{a}(\tau, \nabla u) \mid \operatorname{curl} \operatorname{div} \tau\right)\right| \leq \frac{\varepsilon}{6}\|\nabla u\|_{H^{2}}^{2}+\frac{C(1-\alpha)^{2} \mathrm{We}^{2}}{\varepsilon \alpha^{2}}\|\tau\|_{H^{2}}^{4} .
$$

Substituting these estimates in (4.20), we obtain

$\frac{1-\alpha}{\alpha} \mathrm{We} \frac{d}{d t}\|\operatorname{curl} \operatorname{div} \tau\|_{L^{2}}^{2}+\frac{1}{\alpha}\|\operatorname{curl} \operatorname{div} \tau\|_{L^{2}}^{2} \leq C \alpha \operatorname{Re}^{2}\left\|\nabla u_{t}\right\|_{L^{2}}^{2}+\varepsilon\|\nabla u\|_{H^{2}}^{2}+C_{\varepsilon}\left(\|\nabla u\|_{L^{2}}^{4}+\|\tau\|_{H^{2}}^{4}\right)$.

Putting together (4.19) and (4.21) yields

$\frac{d}{d t}\left(\frac{1-\alpha}{\alpha} \mathrm{We}\left(\|\mathbb{P} \operatorname{div} \tau\|_{L^{2}}^{2}+\|\operatorname{curl} \operatorname{div} \tau\|_{L^{2}}^{2}\right)\right)+\frac{1}{\alpha}\left(\|\mathbb{P} \operatorname{div} \tau\|_{L^{2}}^{2}+\|\operatorname{curl} \operatorname{div} \tau\|_{L^{2}}^{2}\right)$

$$
\leq 2 \varepsilon\|\nabla u\|_{H^{2}}^{2}+\kappa_{3}\left\|\nabla u_{t}\right\|_{L^{2}}^{2}+\|\tau\|_{L^{2}}^{2}+C_{\varepsilon}\left(\|\nabla u\|_{L^{2}}^{4}+\|\tau\|_{H^{2}}^{4}\right),
$$

for some $\kappa_{3}>0$. On the other hand, multiplying equation (4.15) with $\frac{\kappa_{1}+1}{1-\alpha}$ and adding to (4.10) yields $\frac{d}{d t}\left(\mathrm{We}\|\tau\|_{H^{2}}^{2}+\frac{\left(\kappa_{1}+1\right)(2 \operatorname{Re}+1-\alpha)}{1-\alpha}\|\nabla u\|_{L^{2}}^{2}\right)+\frac{\left(\kappa_{1}+1\right) \operatorname{Re}}{1-\alpha}\left\|\partial_{t} u\right\|_{L^{2}}^{2}+\|A u\|_{L^{2}}^{2}+\|\tau\|_{H^{2}}^{2}+2\|\nabla u\|_{H^{2}}^{2}$

$$
\leq \kappa_{4}\left(\|\nabla u\|_{L^{2}}^{2}+\left\|\nabla u_{t}\right\|_{L^{2}}^{2}+\|\mathbb{P} \operatorname{div} \tau\|_{H^{1}}^{2}\right)+C\left(\|A u\|_{L^{2}}^{4}+\|\tau\|_{H^{2}}^{4}+\|\nabla u\|_{L^{2}}^{4}+\|\nabla u\|_{L^{2}}^{6}\right),
$$


for some $\kappa_{4}>0$.

Finally, we estimate $\|\mathbb{P} \operatorname{div} \tau\|_{H^{1}}$ in the right hand side of (4.23). Notice first that in view of the Helmholtz decomposition, we verify that $\operatorname{curl} \operatorname{div} \tau=\operatorname{curl} \mathbb{P} \operatorname{div} \tau$. Moreover, since $\operatorname{div}(\mathbb{P} \operatorname{div} \tau)=0$ and $(\mathbb{P} \operatorname{div} \tau) \cdot \nu=0$, in virtue of Proposition 2.5, there exists a constant $C_{0}$ such that

$$
\|\mathbb{P} \operatorname{div} \tau\|_{H^{1}}^{2} \leq C_{0}\left(\|\mathbb{P} \operatorname{div} \tau\|_{L^{2}}^{2}+\|\operatorname{curl} \operatorname{div} \tau\|_{L^{2}}^{2}\right) .
$$

Then multiplying (4.22) with $\alpha\left(\kappa_{4} C_{0}+1\right)$ and adding to (4.23) implies that

$$
\begin{aligned}
& \frac{d}{d t}\left(\mathrm{We}\|\tau\|_{H^{2}}^{2}+\frac{\left(\kappa_{1}+1\right)(2 \operatorname{Re}+1-\alpha)}{1-\alpha}\|\nabla u\|_{L^{2}}^{2}+(1-\alpha)\left(\kappa_{4} C_{0}+1\right) \text { We }\left(\|\mathbb{P} \operatorname{div} \tau\|_{L^{2}}^{2}+\|\operatorname{curl} \operatorname{div} \tau\|_{L^{2}}^{2}\right)\right) \\
& +\frac{\left(\kappa_{1}+1\right) \operatorname{Re}}{1-\alpha}\left\|\partial_{t} u\right\|_{L^{2}}^{2}+\|A u\|_{L^{2}}^{2}+\|\tau\|_{H^{2}}^{2}+2\|\nabla u\|_{H^{2}}^{2}+\|\mathbb{P} \operatorname{div} \tau\|_{L^{2}}^{2}+\|\operatorname{curl} \operatorname{div} \tau\|_{L^{2}}^{2} \\
\leq & \kappa_{5}\left(\varepsilon\|\nabla u\|_{H^{2}}^{2}+\|\nabla u\|_{L^{2}}^{2}+\left\|\nabla u_{t}\right\|_{L^{2}}^{2}+\|\tau\|_{L^{2}}^{2}\right)+C_{\varepsilon}\left(\|A u\|_{L^{2}}^{4}+\|\tau\|_{H^{2}}^{4}+\|\nabla u\|_{L^{2}}^{4}+\|\nabla u\|_{L^{2}}^{6}\right),
\end{aligned}
$$

for some $\kappa_{5}>0$.

The term $\left\|\nabla u_{t}\right\|_{L^{2}}^{2}$ in the right hand side of above can be absorbed into the left hand side by means of (4.16). Indeed, multiplying (4.16) by $\frac{\kappa_{5}+1}{1-\alpha}$, adding the resulting equation to (4.25) and choosing $\varepsilon=\frac{1-\alpha}{\kappa_{5}(2-\alpha)+1}$, we infer that

$$
\begin{aligned}
& \frac{d}{d t}\left(\mathrm{We}\|\tau\|_{H^{2}}^{2}+\frac{\left(\kappa_{1}+1\right)(2 \operatorname{Re}+1-\alpha)}{1-\alpha}\|\nabla u\|_{L^{2}}^{2}+\frac{\operatorname{Re}\left(\kappa_{5}+1\right)}{1-\alpha}\left\|\partial_{t} u\right\|_{L^{2}}^{2}+\frac{\mathrm{We}\left(\kappa_{5}+1\right)}{2 \alpha(1-\alpha)}\left\|\partial_{t} \tau\right\|_{L^{2}}^{2}+\right. \\
& \left.(1-\alpha)\left(\kappa_{4} C_{0}+1\right) \operatorname{We}\left(\|\mathbb{P} \operatorname{div} \tau\|_{L^{2}}^{2}+\|\operatorname{curl} \operatorname{div} \tau\|_{L^{2}}^{2}\right)\right)+\frac{\operatorname{Re}\left(\kappa_{1}+1\right)}{1-\alpha}\left\|\partial_{t} u\right\|_{L^{2}}^{2}+ \\
& \|A u\|_{L^{2}}^{2}+\|\tau\|_{H^{2}}^{2}+\|\nabla u\|_{H^{2}}^{2}+\left\|\nabla u_{t}\right\|_{L^{2}}^{2}+\frac{\left(\kappa_{5}+1\right)}{\alpha(1-\alpha)}\left\|\partial_{t} \tau\right\|_{L^{2}}^{2}+\|\mathbb{P} \operatorname{div} \tau\|_{L^{2}}^{2}+\|\operatorname{curl} \operatorname{div} \tau\|_{L^{2}}^{2} \\
\leq & C\left(\|A u\|_{L^{2}}^{4}+\|\tau\|_{H^{2}}^{4}+\|\nabla u\|_{L^{2}}^{4}+\|\nabla u\|_{L^{2}}^{6}+\left\|\partial_{t} u\right\|_{L^{2}}^{4}+\left\|\partial_{t} \tau\right\|_{L^{2}}^{4}\right)+\kappa_{6}\left(\|\nabla u\|_{L^{2}}^{2}+\|\tau\|_{L^{2}}^{2}\right),
\end{aligned}
$$

for some $\kappa_{6}>0$. Finally, using the assumption $0<\alpha<1$ and multiplying (4.11) with $\frac{\kappa_{6}+1}{1-\alpha}$ and adding it to 4.25 yields

$$
\begin{aligned}
& \frac{d}{d t}\left(\frac{\operatorname{Re}\left(\kappa_{6}+1\right)}{1-\alpha}\|u\|_{L^{2}}^{2}+\frac{\mathrm{We}\left(\kappa_{6}+1\right)}{2 \alpha(1-\alpha)}\|\tau\|_{L^{2}}^{2}+\mathrm{We}\|\tau\|_{H^{2}}^{2}+\frac{\left(\kappa_{1}+1\right)(2 \operatorname{Re}+1-\alpha)}{1-\alpha}\|\nabla u\|_{L^{2}}^{2}\right. \\
& +\frac{\operatorname{Re}\left(\kappa_{5}+1\right)}{1-\alpha}\left\|\partial_{t} u\right\|_{L^{2}}^{2}+\frac{\mathrm{We}\left(\kappa_{5}+1\right)}{2 \alpha(1-\alpha)}\left\|\partial_{t} \tau\right\|_{L^{2}}^{2}+(1-\alpha)\left(\kappa_{4} C_{0}+1\right) \mathrm{We}\left(\|\mathbb{P} \operatorname{div} \tau\|_{L^{2}}^{2}+\right. \\
& \left.\left.\|\operatorname{curl} \operatorname{div} \tau\|_{L^{2}}^{2}\right)\right)+\frac{\operatorname{Re}\left(\kappa_{1}+1\right)}{1-\alpha}\left\|\partial_{t} u\right\|_{L^{2}}^{2}+\|A u\|_{L^{2}}^{2}+\|\tau\|_{H^{2}}^{2}+\|\nabla u\|_{H^{2}}^{2}+\left\|\nabla u_{t}\right\|_{L^{2}}^{2} \\
& +\frac{\left(\kappa_{5}+1\right)}{\alpha(1-\alpha)}\left\|\partial_{t} \tau\right\|_{L^{2}}^{2}+\|\nabla u\|_{L^{2}}^{2}+\|\tau\|_{L^{2}}^{2}+\|\mathbb{P} \operatorname{div} \tau\|_{L^{2}}^{2}+\|\operatorname{curl} \operatorname{div} \tau\|_{L^{2}}^{2} \\
& \leq C\left(\|A u\|_{L^{2}}^{4}+\|\tau\|_{H^{2}}^{4}+\|\nabla u\|_{L^{2}}^{4}+\|\nabla u\|_{L^{2}}^{6}+\left\|\partial_{t} u\right\|_{L^{2}}^{4}+\left\|\partial_{t} \tau\right\|_{L^{2}}^{4}\right) .
\end{aligned}
$$

After all these calculations, we are glad to define the functions $F:[0, \infty) \rightarrow \mathbb{R}, G:[0, \infty) \rightarrow \mathbb{R}$ and $H:[0, \infty) \rightarrow \mathbb{R}$ as

$$
\begin{aligned}
F(t):= & (1-\alpha)\left(\kappa_{4} C_{0}+1\right) \mathrm{We}\left(\|\mathbb{P} \operatorname{div} \tau\|_{L^{2}}^{2}+\|\operatorname{curl} \operatorname{div} \tau\|_{L^{2}}^{2}\right) \\
& +\frac{\operatorname{Re}\left(\kappa_{6}+1\right)}{1-\alpha}\|u\|_{L^{2}}^{2}+\frac{\mathrm{We}\left(\kappa_{6}+1\right)}{2 \alpha(1-\alpha)}\|\tau\|_{L^{2}}^{2}+\mathrm{We}\|\tau\|_{H^{2}}^{2} \\
& +\frac{\left(\kappa_{1}+1\right)(2 \operatorname{Re}+1-\alpha)}{1-\alpha}\|\nabla u\|_{L^{2}}^{2}+\frac{\operatorname{Re}\left(\kappa_{5}+1\right)}{1-\alpha}\left\|\partial_{t} u\right\|_{L^{2}}^{2} \\
& +\frac{\mathrm{We}\left(\kappa_{5}+1\right)}{2 \alpha(1-\alpha)}\left\|\partial_{t} \tau\right\|_{L^{2}}^{2},
\end{aligned}
$$


and

$$
\begin{aligned}
G(t):= & \frac{\operatorname{Re}\left(\kappa_{1}+1\right)}{1-\alpha}\left\|\partial_{t} u\right\|_{L^{2}}^{2}+\|A u\|_{L^{2}}^{2}+\|\tau\|_{H^{2}}^{2}+\|\nabla u\|_{H^{2}}^{2}+\left\|\nabla u_{t}\right\|_{L^{2}}^{2} \\
& +\frac{\left(\kappa_{5}+1\right)}{\alpha(1-\alpha)}\left\|\partial_{t} \tau\right\|_{L^{2}}^{2}+\|\nabla u\|_{L^{2}}^{2}+\|\tau\|_{L^{2}}^{2}+\|\mathbb{P} \operatorname{div} \tau\|_{L^{2}}^{2}+\|\operatorname{curl} \operatorname{div} \tau\|_{L^{2}}^{2}
\end{aligned}
$$

and

$$
H(t):=\left\|\partial_{t} u\right\|_{L^{2}}^{2}+\|A u\|_{L^{2}}^{2}+\|\tau\|_{H^{2}}^{2}+\left\|\partial_{t} \tau\right\|_{L^{2}}^{2}+\|\nabla u\|_{L^{2}}^{2}+\|\nabla u\|_{L^{2}}^{4} .
$$

We now rewrite (4.25) as an inequality of the form

$$
\frac{d}{d t} F(t)+G(t) \leq C H(t) G(t)
$$

and estimate $H(t)$ in terms of $F(t)$. To this end, observe that by (4.3), (4.12) and (4.8) we deduce that

$$
\|A u\|_{L^{2}}^{2} \leq C\left(\|\nabla u\|_{L^{2}}^{2}+\left\|\partial_{t} u\right\|_{L^{2}}^{2}+\|\mathbb{P} \operatorname{div} \tau\|_{L^{2}}^{2}+\|\nabla u\|_{L^{2}}^{6}\right) .
$$

Hence, there exists a constant $M_{1}=M_{1}(\operatorname{Re}$, We,$\alpha)>0$ such that

$$
H(t) \leq M_{1}\left(F(t)+F(t)^{2}+F(t)^{3}\right), \quad t \geq 0 .
$$

Substituting (4.28) into (4.26), we get

$$
\frac{d}{d t} F(t)+\left(1-C M_{1}\left(F(t)+F(t)^{2}+F(t)^{3}\right)\right) G(t) \leq 0, \quad t \geq 0 .
$$

We are now finally in the position to estimates $F(t)$. In order to do so, define $\delta_{0}>0$ small enough, such that $\delta_{0}+\delta_{0}^{2}+\delta_{0}^{3}<\frac{1}{2 C M_{1}}$, where $C$ is the constant appearing in (4.29).

Assume that the differential inequality (4.29) holds for all $t \geq 0$ and $F$ being absolutely continuous and $G$ being nonnegative. Then

$$
F(t)<\delta_{0} \text { for all } t \geq 0 \text { provided } F(0)<\delta_{0} .
$$

Assume that this assertion were not true. Let $t_{1}>0$ be the first time where $F(t) \geq \delta_{0}$. Then

$$
F\left(t_{1}\right)=\delta_{0} \text { and } F(t)<\delta_{0} \text { for all } 0 \leq t<t_{1} .
$$

Consequently, for all $0 \leq t \leq t_{1}$,

$$
1-C M_{1}\left(F(t)+F(t)^{2}+F(t)^{3}\right) \geq 1-C M_{1}\left(\delta_{0}+\delta_{0}^{2}+\delta_{0}^{3}\right)>\frac{1}{2} .
$$

Assertion (4.29) implies now that

$$
\frac{d}{d t} F(t)+\frac{1}{2} G(t) \leq 0 \text { for all } 0 \leq t \leq t_{1} .
$$

Integrating (4.32) from 0 to $t_{1}$, we obtain

$$
F\left(t_{1}\right)+\frac{1}{2} \int_{0}^{t_{1}} G(s) d s \leq F(0)<\delta_{0},
$$

which contradicts (4.31). Thus (4.30) holds true.

Given this fact, the proof of Theorem 1.1 can now be finished easily. In fact, let $T^{\star}$ be the lifespan of the local solution $(u, p, \tau)$ given in Proposition 3.1. Assuming that (1.5) holds with $\varepsilon_{0}$ to be determined below, we verify that

$$
F(0) \leq C\left(\left\|u_{0}\right\|_{H^{1}}^{2}+\left\|\tau_{0}\right\|_{H^{2}}^{2}+\left\|u_{t}(0)\right\|_{L^{2}}^{2}+\left\|\tau_{t}(0)\right\|_{L^{2}}^{2}\right) \leq C\left(\varepsilon_{0}^{2}+\varepsilon_{0}^{4}\right) .
$$

Choose now $\varepsilon_{0}$ such that $C\left(\varepsilon_{0}^{2}+\varepsilon_{0}^{4}\right)<\delta_{0}$. Then, in virtue of (4.30) and the proof of (4.33), we obtain

$$
\sup _{0 \leq t \leq T^{\star}} F(t)+\frac{1}{2} \int_{0}^{T^{\star}} G(t) d t<\delta_{0} .
$$


In particular, it follows from the definition of $F(t)$ and $G(t)$, (4.34), (4.27) and (4.8) that

$$
\begin{aligned}
& \sup _{0 \leq t \leq T^{\star}}\left(\|u(t)\|_{D(A)}^{2}+\left\|u^{\prime}(t)\right\|_{L^{2}}^{2}+\|\tau(t)\|_{H^{2}}^{2}+\left\|\tau^{\prime}(t)\right\|_{L^{2}}^{2}\right) \\
& +\int_{0}^{T^{\star}}\left(\|\nabla u(t)\|_{H^{2}}^{2}+\left\|\nabla u^{\prime}(t)\right\|_{L^{2}}^{2}+\|\tau(t)\|_{H^{2}}^{2}+\left\|\tau^{\prime}(t)\right\|_{L^{2}}^{2}\right) d t \leq C .
\end{aligned}
$$

We thus deduce that the local solution $(u, p, \tau)$ can be extended for all positive times. This completes the proof of Theorem 1.1

Acknowledgments This work was carried out while the first and the third authors are visiting the Department of Mathematics at the Technical University of Darmstadt. They would express their gratitude to Prof. Matthias Hieber for his kind hospitality and the Deutsche Forschungsgemeinschaft (DFG) for financial support.

We would like thank Paolo Galdi for stimulating discussion concerning Oldroyd-B fluids and the third author also would like to thank Tobias Hansel for his sincere help.

Daoyuan Fang and Ruizhao Zi were partially supported by NSFC 10931007 and ZNSFC Z6100217.

\section{REFERENCES}

[1] J. Y. Chemin, N. Masmoudi, About lifespan of regular solutions of equations related to viscoelastic fluids. SIAM J. Math. Anal, 33(2001), 84-112.

[2] E. Fernández-Cara, F. Guillén, R. Ortega, Some theoretical results concerning non-Newtonian fluids of the Oldroyd kind. Ann. Scuola Norm. Sup. Pisa, 26(1998), 1-29.

[3] G.P. Galdi, An introduction to the Mathematical Theory of the Navier-Stokes Equations, Vol I, Springer-Verlag, New York, 1994.

[4] V. Girault, P. A. Raviart, Finite Elements Methods for Navier-Stokes Equations: Theory and Algorithms, SpringerVerlag, Berlin, 1986.

[5] C. Guillopé, J. C. Saut, Existence results for the flow of viscoelastic fluids with a differential constitutive law. Nonlinear Anal. 15(1990), 849-869.

[6] M. Hieber, Y. Naito, Y. Shibata, Global existence results for Oldroyd-B fluids in exterior domains. J. Diff. Eq. 252 (2012), 2617-2629.

[7] O. Kreml, M. Pokorny, On the local strong solutions for a system describing the flow of a viscoelastic fluid. In: Nonlocal and Abstract Parabolic Equations and their Applications, Banach Center Publ., 86, Polish Acad. Sci. Inst. Math., Warsaw, (2009), 195-206.

[8] Z. Lei, Global existence of cloassical solutions for some Oldroyd-B model via the incompressible limit. Chinese Ann. Math. 27(2006), 565-580.

[9] Z. Lei, On 2D viscoelasticity with small strain. Arch. Ration. Mech. Anal. 198(2010), 13-37.

[10] Z. Lei, C. Liu and Y. Zhou, Global solutions for incompressible viscoelastic fluids. Arch. Ration. Mech. Anal. 188(2008), 371-398.

[11] Z. Lei, N. Masmoudi, Y. Zhou, Remarks on the blowup criteria for Oldroyd models. J. Diff. Equ. 248(2010), 328-341.

[12] Z. Lei, Y. Zhou, Global existence of classical solutions for the two-dimensional Oldroyd model via the incompressible limit. SIAM J. Math. Anal. 37(2005), 797-814.

[13] F. H. Lin, C. Liu and P. Zhang, On hydrodynamics of viscoelastic fluids. Comm. Pure Appl. Math. 58(2005), $1437-1471$.

[14] F. H. Lin, P. Zhang, On the initial-boundary value problem of the incompressible viscoelastic fluid system. Comm. Pure Appl. Math. 61(2008), 539-558.

[15] P. L. Lions, N. Masmoudi, Global solutions for some Oldroyd models of non-Newtonian flows. Chinese Ann. Math. Ser. B, 21(2000), 131-146.

[16] L. Molinet, R. Talhouk, On the global and periodic regular flows of viscoelastic fluids with a differential constitutive law. Nonlinear Diff. Equations Appl. 11(2004), 349-359.

[17] J. G. Oldroyd, Non-Newtonian effects in steady motion of some idealized elastico-viscous liquids. Proc. Roy. Soc. London. 245(1958), 278-297.

[18] R. Talhouk, Existence locale et unicité d'écoulements de fluides viscoélastiques dans des domaines non bornés. [Local existence and uniqueness of viscoelastic fluid flows in unbounded domains] C. R. Acad. Sci. Paris 328 (1999), 87-92. 
Department of Mathematics, Zhejiang University, Hangzhou 310027, China

Technische Universität Darmstadt, Fachbereich Mathematik, Schlossgartenstr. 7, D-64289 Darmstadt, Germany and

Center of Smart Interfaces, Petersenstr. 32, D-64287 Darmstadt

Department of Mathematics, Zhejiang University, Hangzhou 310027, China

E-mail address: dyf@zju.edu.cn

E-mail address: hieber@mathematik.tu-darmstadt.de

E-mail address: ruizhao3805@163.com 